\title{
Lubrication with Oil-Compatible Polymer Brushes
}

\section{Journal Article}

Author(s):

Bielecki, Robert M.; Benetti, Edmondo M.; Kumar, Deepak; Spencer, Nicholas D.

Publication date:

2012

Permanent link:

https://doi.org/10.3929/ethz-b-000046212

Rights / license:

In Copyright - Non-Commercial Use Permitted

Originally published in:

Tribology Letters 45(3), https://doi.org/10.1007/s11249-011-9903-6 


\title{
Lubrication with Oil-Compatible Polymer Brushes
}

\author{
Robert M. Bielecki • Edmondo M. Benetti • \\ Deepak Kumar • Nicholas D. Spencer
}

Received: 9 November 2011 / Accepted: 19 December 2011/Published online: 8 January 2012

(C) Springer Science+Business Media, LLC 2012

\begin{abstract}
A polymer-brush-based, surface-modification strategy for friction and wear reduction in hard contact under boundary-lubrication conditions is proposed, specifically for a non-aqueous environment. Surface-initiated atom-transfer radical polymerization was employed for the synthesis of three different oil-compatible, hydrophobic polymer brushes based on alkyl methacrylates. This study presents polymerization kinetics, chemical characterization by means of Fourier-transform infrared spectroscopy and surface morphologies observed in atomic force microscopy. The lubrication properties of the anchored polymers were evaluated macroscopically by means of ball-on-disk methods and on the nanonewton scale by lateral force microscopy and showed significant reduction in friction up to contact pressures as high as $460 \mathrm{MPa}$. The frictional response of surface-grafted polymers is shown to depend strongly on the compatibility of the polymer with the chosen lubricating fluid.
\end{abstract}

Keywords Polymer brush - Surface modification · ATRP · Oil lubrication

\section{Introduction}

Densely grafted, surface-tethered polymers in the presence of a good solvent attain a brush-like, stretched

Electronic supplementary material The online version of this article (doi:10.1007/s11249-011-9903-6) contains supplementary material, which is available to authorized users.

R. M. Bielecki · E. M. Benetti · D. Kumar · N. D. Spencer ( $\square)$ Laboratory for Surface Science and Technology,

Department of Materials, ETH Zurich, Wolfgang-Pauli-Strasse

10, HCI G517, 8093 Zurich, Switzerland

e-mail: nspencer@ethz.ch configuration [1, 2]. Klein [3] and others [4-6] have investigated frictional properties of polymer-brush-modified surfaces and found that when immersed in a good solvent they lead to a substantial decrease in frictional forces, resulting from the fluid-like interfacial layer $[3,7]$. The friction-reduction mechanism for solvated polymer brushes is attributed (a) to repulsive terms consisting of the osmotic pressure within the brush system plus the resistance to interpenetration of the grafted chains, combined with (b) the confinement of the lubricant within the contact area and (c) the formation of a low-shear interface in the outer reaches of the brush, where solvent concentration is the highest [8].

The behavior of polymer brushes under tribological stress depends on the way in which they are anchored to surface, their chemical and physical state under the liquid, and the type and level of mechanical stresses within the contact zone. At contact pressures below $1 \mathrm{MPa}$ a stable coefficient of friction below 0.01 can be maintained for thousands of rotations (sliding distance over $350 \mathrm{~m}$ ) in macroscopic pin-on-disk measurements with poly(methacrylic acid) brushes in water [4]. Polymer brushes grafted to surfaces by means of electrostatic interactions have been reported to maintain a coefficient of friction of 0.1 under contact pressures of several hundreds of MPa [6].

Developments in polymer chemistry, especially in controlled radical polymerization, have led to effective methods for the synthesis of surface-grafted polymer chains $[9,10]$. This approach, also known as "grafting from", yields polymer-brush coatings of a higher macromolecular chain density in comparison to "grafting to" of the bulky polymer chains [11]. Grafting-from coatings are covalently attached to the substrate, and thus potentially more solvent resistant and mechanically stable. Sakata et al. [12] have shown that surface-grafted, ATRP-polymerized poly(methyl methacrylate) 
brushes can be used at contact pressures of $200 \mathrm{MPa}$ to maintain the coefficient of friction as low as 0.05 , when in the presence of a good solvent.

Surface-initiated atom-transfer radical polymerization (SI-ATRP) is a highly versatile method for controlled radical polymerization, and has proven to be successful in polymerising many different monomers with a wide variety of reactivities [13-15]. Its principle relies on a dynamic equilibrium between the dormant and active chain ends, which is strongly shifted to the dormant state, thus diminishing the effects of undesired termination. In a typical reaction setup, transition-metal halides, frequently copper bromides or chlorides, rendered soluble by nitrogen-based ligands, are constantly undergoing oxidation and reduction to form a radical at the chain end. This allows the addition of a monomer, followed by an immediate return to the halide-terminated deactivated state. The rate of propagation in ATRP can be controlled by adjusting the monomer concentration and the ratio of the activator/deactivator species [16]. The polarity and the amount of solvent used in the reaction, together with the redox potential of the formed transition-metal complex can strongly affect the polymerization reaction $[9,17,18]$.

The use of surface-grafted polymers to enhance lubricity has been reported extensively in the presence of aqueous [12, 19-21] lubricants, but sparsely [5, 22] for non-aqueous (e.g., oil) systems. The combination of a polymer brush with an oil has the potential to combine the excellent boundary-lubrication properties of brushes with the rheological and high-temperature advantages of oil. Hydrophobic polymer brushes under oil can provide a thick, soft, swollen polymer layer that prevents asperity-asperity contact between the mating surfaces. In this situation, a low-shear-strength plane at the contact zone is expected [3]. A covalently tethered polymer brush, when immersed in a good solvent for the polymer, will effectively become a soft lubricant reservoir. The use of polymethacrylatebased viscosity-index improvers as friction-reducing oil additives has been studied by Fan et al. [23]. They concluded that the friction-reducing effect occurs particularly for polymers containing blocks of surface-active monomers in their structures, which lead to the formation of a "brushlike" film on the surface. Such a polymeric layer was found to support load and separate the opposing surfaces-even under stationary conditions.

While there have been several recent reports of the use of "grafting from" to place molecular brushes containing long side chains on surfaces, most of these have involved oligo or poly(ethylene glycol) (OEG or PEG) polymethacrylates. These layers have found interesting applications in aqueous systems and in particular for the design of biological platforms $[24,25]$. Very few reports exist in the literature of the surface-initiated polymerization of

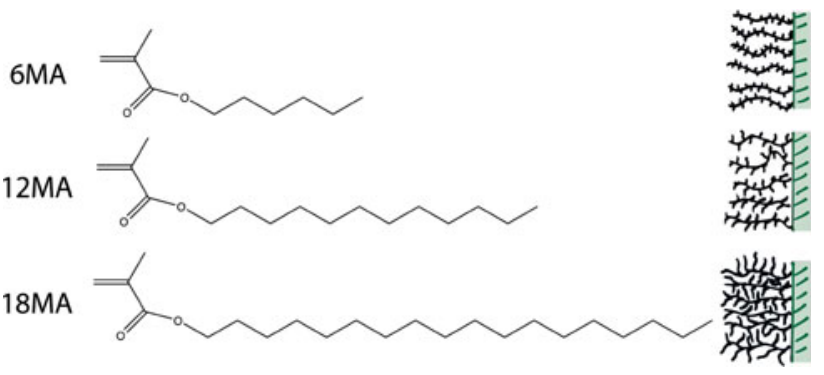

Fig. 1 Chemical structures of the monomers used in this study hexyl methacrylate (6MA), dodecyl methacrylate (12MA), and octadecyl methacrylate (18MA). On the right, schematically presented bottlebrush-like structures of the corresponding polymer coatings

hydrophobic polymers based on longer-chain alkyl methacrylates (6-20 side carbons) to form molecular brushes.

While hydrophobic polymers with long alkyl side chains have found several applications in bulk or solution systems as elastomers and surfactants, they have never been applied to modify the physicochemical properties of surfaces and materials. Such polymers present intriguing properties, such as crystallizable side chains and low melting points and glass-transition temperatures, which make them interesting candidates as surface modifiers for materials designed for contact with non-polar media.

Previously, such polymers have mainly been prepared in solution, and via free-radical polymerization [26]. In this study we focused on the synthesis of alkyl-chain-substituted polymethacrylate brush systems: octadecyl methacrylate (also known as stearyl), dodecyl methacrylate (also known as lauryl), and hexyl methacrylate (designated as P18MA, P12MA, and P6MA, respectively) (Fig. 1). These have been prepared by SI-ATRP from silicon surfaces, and their physicochemical and tribological properties characterized under good- and bad-solvent conditions.

\section{Experimental}

\subsection{Materials}

Dimethylchlorosilane (Aldrich-Fine Chemicals, 98\%), 10-undecen-1-ol (Aldrich-Fine Chemicals, 98\%), 2-bromo2-methylpropionyl bromide (Acros Organics, 98\%), and chloroplatinic acid hexahydrate (ABCR Deutschland 99.9\%) were used for initiator synthesis as received.

Monomers, hexyl methacrylate (Aldrich-Fine Chemicals, 98\%), dodecyl methacrylate (Acros Organics, 96\%), and octadecyl methacrylate (TCI Deutschland GmbH, $>95 \%$ ) were diluted with pentane and purified from MEHQ inhibitor by passing through an alumina column.

4,4'-Dinonyl-2,2'-bipyridine (Aldrich-Fine Chemicals, 97\%), anisole (Acros Organics, 99\%), and copper(II) 
bromide (Sigma-Aldrich, 99\%) were used as received. Copper bromide (Aldrich-Fine Chemicals, $5 \mathrm{~N}$ ) was purified by overnight washing in glacial acetic acid, washing with methanol and diethyl ether and drying under vacuum before use.

\subsection{Synthesis of 11-(2-Bromo-2-methyl-propionyl)- dimethylchlorosilane Initiator (BPCS)}

The synthesis of the initiator molecules followed a protocol described by Sanjuan et al. [13]. To $10.7 \mathrm{~mL}$ of 10-undecen-1-ol in $50 \mathrm{~mL}$ dry THF (98\% Sigma Aldrich), were added $9 \mathrm{~mL}(60 \mathrm{mmol})$ triethylamine $(99.5 \%$ Sigma Aldrich), followed by dropwise addition of a solution of $7 \mathrm{~mL}$ bromoisobutyrate in $20 \mathrm{~mL}$ dry THF. The mixture was stirred under argon for $24 \mathrm{~h}$ and diluted with $100 \mathrm{~mL}$ of hexane (the hexane remains on top), washed twice with $100 \mathrm{~mL}$ of $2 \mathrm{M} \mathrm{HCl}(42 \mathrm{~mL}$ of $37 \% \mathrm{HCl}$ added to $208 \mathrm{~mL}$ $\mathrm{H}_{2} \mathrm{O}$ ) and 4 times with $100 \mathrm{~mL}$ of ultra-pure water. The organic phase was dried over magnesium sulfate for $60 \mathrm{~min}$, and filtered through paper prior to concentration at $130 \mathrm{mbar}$ at $40{ }^{\circ} \mathrm{C}$. The un-reacted hydroxyl-terminated olefins were removed by passing through a silica gel 60 column. The colorless oily product was concentrated under vacuum with rotation, and stored under $\mathrm{Ar}$ at $4{ }^{\circ} \mathrm{C}$ until needed. In the second reaction step, $2.54 \mathrm{~g}$ of the 10 -undecen-1-yl 2-bromo-2-methylpropionate were added to approx. $10 \mathrm{mg}$ of the platinum catalyst and $7.93 \mathrm{~mL}$ of dimethylchlorosilane. The compounds were stirred under Ar overnight in the dark and then filtered through a silica plug. The excess of unreacted silane was removed under vacuum. The synthesis of the end product was confirmed by ${ }^{1} \mathrm{H}$ NMR.

\subsection{BPCS Surface Functionalization}

Silicon wafers (P/B $\langle 100\rangle$, Si-Mat Silicon Wafers, Germany) were cut into $20 \times 20 \mathrm{~mm}^{2}$ pieces, washed three times with 2-isopropanol in a sonication bath, and treated for $30 \mathrm{~min}$ in the UV Ozone cleaner (UV/Ozone ProCleaner ${ }^{\mathrm{TM}}$ and ProCleaner $^{\mathrm{TM}}$ Plus, BioForce, IA, USA). Cleaned wafers were immediately immersed in a $10 \mathrm{mM}$ solution of the BPCS initiator in freshly distilled toluene and incubated under an Ar atmosphere for $3 \mathrm{~h}$. This was followed by washing 5 times in toluene and brief sonication in fresh toluene to remove the weakly bound molecules, before rinsing again with 2 -isopropanol. Samples prepared in this way were dried in a stream of nitrogen and stored under a $\mathrm{N}_{2}$ atmosphere until used. The choice of a monofunctional silane was dictated by the lower probability of multilayer formation, which may occur in the case of trichloro or tri methoxy silanes.

\subsection{SI-ATRP of Poly(alkyl methacrylates)}

Polymerization reactions were carried out in an oxygen-free Schlenk line under argon. Monomers were mixed with anisole in the ratio of 9:1, dNbpy was added and the flask plugged with a rubber septum. After stirring and dissolution of the ligand, the solution underwent four freeze-pump-thaw cycles, to be later transferred to a second flask containing copper bromides. The solution containing copper bromide, monomer, and ligand was stirred for $5 \mathrm{~min}$ in a hot oil bath to achieve a darkbrown homogeneous mixture, which was later transferred with an oxygen-free syringe to tubes containing initiatorfunctionalized samples. For the polymerization results presented below, the monomer:ligand:copper(I) bromide molar ratio was maintained constant for all polymers at 150:1.5:1. The reactions were carried out at $110^{\circ} \mathrm{C}$ under $\mathrm{Ar}$ and quenched by opening to air and addition of toluene to dilute the reaction mixture.

Afterwards, samples were rinsed thoroughly with toluene and a $0.1 \mathrm{M}$ solution of $\mathrm{Na}_{2}$ EDTA was added to remove the remaining bromide moieties, followed by another toluene-washing step.

\subsection{Ellipsometry}

A variable-angle spectroscopic ellipsometer (VASE) (M-2000F, LOT Oriel GmbH, Darmstadt, Germany) was used to determine the reaction kinetics non-destructively, via the dry thickness of the surface-bound polymers. The ellipsometric measurement was collected at three different angles of incidence $65^{\circ}, 70^{\circ}$, and $75^{\circ}$, and the incident wavelength was varied between 995 and $370 \mathrm{~nm}$. The obtained thickness values are a result of a fit to a threelayer model, $\mathrm{Si}$ jell/ $\mathrm{SiO}_{2} /$ Cauchy, defined in the WVASE32 software (LOT Oriel GmbH, Darmstadt, Germany).

\subsection{Polymer-Brush Characterization}

The brush films were studied with transmission Fouriertransform infrared (FTIR) spectroscopy. The data was obtained in transmission mode on a Bruker IFS 66v IR, with a liquid-nitrogen-cooled MCT detector. The film thickness for all coatings analysed in FTIR was ca. $120 \mathrm{~nm}$. Collected spectra were baseline corrected and normalized with respect to the peak found at around $1730 \mathrm{~cm}^{-1}$ —characteristic for $\mathrm{C}=\mathrm{O}$ vibration stretching and typical of esters.

\subsection{Atomic Force Microscopy: Pull-Off Force Measurements and Lateral-Force Microscopy (LFM)}

All pull-off (adhesive) and lateral (friction) forces were measured on the nanoscale with an MFP-3D atomic force 
microscope (AFM) (Asylum Research, Santa Barbara, CA). The measurements were carried out in $n$-hexadecane (to simulate an oil-like medium) and ethanol media. Colloidal probes [27] were prepared by mounting $\mathrm{SiO}_{2}$ (silica) colloidal spheres (Kromasil, Brewster, NY, diameter = $15 \pm 0.5 \mu \mathrm{m}$ ) onto tipless silicon cantilevers (Micromash, San Jose, CA) by means of a UV-curable adhesive. The normal stiffness $(0.139,0.17,0.246$, and $0.342 \mathrm{~N} / \mathrm{m})$ of the cantilevers was found by means of the thermal-tuning technique, incorporated into the AFM software. Pull-off and lateral forces were measured through deflection of a laser beam on the position-sensitive photodiode (PSPD). Normal and lateral sensitivities were used to convert the PSPD generated signal (in volts) into Newtons for normaland lateral-force measurements, respectively. The normal sensitivity of the colloidal probe was measured by determining the slope of the curve when a colloidal probe is pressed against a hard $\left(\mathrm{SiO}_{2}\right)$ surface. Lateral sensitivity was determined by pressing the colloidal probe laterally against a large hard sphere and measuring the deflection of the cantilever, as proposed by Cannara et al. [28]. The frictional response reported here for each load is the average of 20-30 line scans of $5 \mu \mathrm{m}$ at a minimum of three different locations. The friction force for each scan is calculated by averaging the forward and reverse friction forces as (friction force forward-friction force reverse)/2.

The swelling ratios of the polymer brushes were obtained from step-height measurements of intentionally scratched coatings. Step measurement was performed under very low forces $(2 \mathrm{nN})$ using highly sensitive $40-\mu \mathrm{m}-$ long low-stiffness cantilevers in tapping mode (BLAC40TS-C2, Olympus, Japan, $k=0.09 \mathrm{~N} / \mathrm{m}, f=110 \mathrm{kHz}$ in air, tip radius $10 \mathrm{~nm}$ ). Samples were measured under ambient conditions (dry state), immersed in ethanol and in hexadecane. Values of at least ten different step-profiles were averaged. The swelling ratio was calculated as the step height under a given liquid divided by a value measured in dry state.

\subsection{Ball-on-Disk Tribological Test}

Macroscopic pin-on-disk experiments were performed on a Newton scale, by means of a CETR Tribometer (UMT-2, Center for Tribology, Campbell CA, USA). Measurements were made at room temperature.

The applied load was monitored at all times with a load cell and maintained at $3 \mathrm{~N}$. The cup-shaped sample holder was specially designed with a $20 \times 20 \mathrm{~mm}^{2}$ machined undercut, to allow for a secure fixation of the silicon wafer. Highly polished Si balls, $6 \mathrm{~mm}$ in diameter (J. Hauser $\mathrm{GmbH}$, Solms, Germany, $R_{\mathrm{a}}$ below $20 \mathrm{~nm}$ ) were mounted in a stainless steel holder. The contact pair was completely immersed in the lubricant by filling the cup with hexadecane. 11 speed values were selected between 0.002 and $1.5 \mathrm{~cm} \mathrm{~s}^{-1}$. At each speed, coefficient-of-friction values were averaged over 20 revolutions.

\section{Results}

\subsection{Polymerization of Alkyl Methacrylates}

The BPCS-initiator-functionalized surfaces were characterized by static-contact-angle (CA) measurements. BPCS layers adsorbed on a UV/ozone-cleaned ultra-hydrophilic surface (initial contact angle below $3^{\circ}$ ) resulted in contact angles of $77^{\circ} \pm 2^{\circ}$. The degree of hydrophilicity is a useful assay for the estimation of self-assembled monolayer (SAM) composition in the case of mixed monolayers, or to identify less densely packed structures, in the case of single-component SAMs on silicon [29, 30]. The ellipsometric thickness of the BPCS layer was measured as $1.8 \pm$ $0.1 \mathrm{~nm}$, which would correspond well to the estimated thickness of a compact and tilted $\left(28^{\circ}\right)$ SAM structure.

The polymerization kinetics are presented as averaged dry film-thickness values, measured at five different spots on each sample. The variation of dry film thickness for three different polymers as a function of the polymerization time is shown in Fig. 2. It was possible to achieve coatings thicker than $100 \mathrm{~nm}$ for all three alkyl methacrylates; nonetheless, dodecyl methacrylate (P12MA) was polymerized more readily than the other two monomers, reaching a maximum dry thickness of nearly $350 \mathrm{~nm}$ after $3 \mathrm{~h}$ of polymerization.

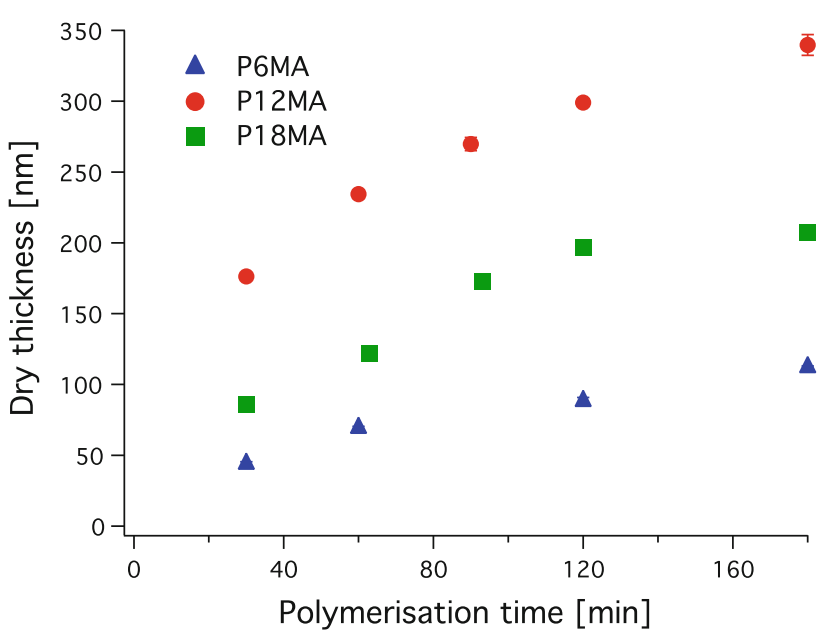

Fig. 2 Polymer-brush thickness (dry, measured by ex situ ellipsometry) as a function of polymerization time. Temperature $110^{\circ} \mathrm{C}$, with 10 vol.\% of anisole as a solvent and 150:1.5:1 monomer:ligand:copper (I) ratio. The $\mathrm{Cu}(\mathrm{II}) / \mathrm{Cu}(\mathrm{I})$ ratio was maintained at 0.15 


\subsection{FTIR Spectra}

Figure 3 shows the transmission FTIR spectra for P18MA, P12MA, and P6MA films of similar thickness, in a dry state. The characteristic absorption bands between 2,950 and $2,840 \mathrm{~cm}^{-1}$ are assigned to $\mathrm{CH}_{3}$ and $\mathrm{CH}_{2}$. The intensity of the $\mathrm{CH}_{3}$ peaks (at ca. 2,955 and $2,872 \mathrm{~cm}^{-1}$ ) is approximately equal for the three polymer brushes, whilst for the $\mathrm{CH}_{2}$ peaks (at ca. 2,925 and $2,850 \mathrm{~cm}^{-1}$ ), an increasing peak area is observed as the length of the sidechains on the methacrylates increases. The $\mathrm{CH}_{2}$ stretching vibration bands are shifted from $2,859 \mathrm{~cm}^{-1}$, via 2,854 to $2,850 \mathrm{~cm}^{-1}$, and from $2,919 \mathrm{~cm}^{-1}$, via 2,925 to $2,931 \mathrm{~cm}^{-1}$ for the hexyl-, dodecyl- and octadecyl-based polymer series, respectively, which may be attributed to their different degrees of molecular aggregation. For all three polymers, $\mathrm{C}=\mathrm{O}$ stretching vibration peaks were found at $1,733 \mathrm{~cm}^{-1}$. Further, peaks at around $1,470 \mathrm{~cm}^{-1}$ were assigned to $\mathrm{C}-\mathrm{H}$ deformations, with increasing intensity with increasing side-chain length.

\subsection{Surface Morphology of Dried Polymer Coatings and Swelling-Ratio Measurements}

The surface morphology of the toluene-treated and dried polymer-coated samples was studied at room temperature $\left(24{ }^{\circ} \mathrm{C}\right)$ in ambient air. Images recorded with AFM reveal differences between the morphology of the different samples (see Supplementary Material). It should be borne in mind, however, that while the surface roughness and surface morphology of the dried polymer brushes may be of general interest for these polymers, they will play a

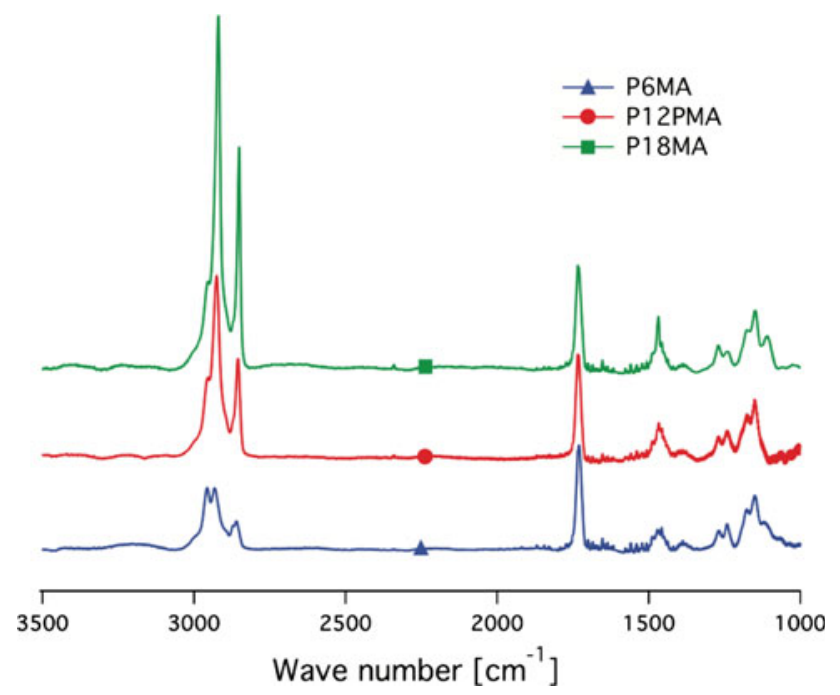

Fig. 3 Transmission-mode FTIR absorbance spectra of dry poly(alkyl methacrylate) brushes on a silicon wafer minimal role in determining the coefficient of friction for polymer brushes immersed in a good solvent $[22,31]$.

The height change of the polymer brush upon immersion in a solvent was measured by low-force tapping-mode imaging of steps obtained by local coating removal. All three polymer brushes, when immersed in ethanol, have a negligible thickness change, since they are not soluble and do not swell. The two longer-side-chain polymer brushes when immersed in hexadecane increase their height and present up to ninefold increases in the step heights for the P12MA. The P6MA polymer brush does not swell in hexadecane.

\subsection{Tribological Response of Surface-Initiated Alkyl Methacrylate Polymers}

To study the lubricating properties of the proposed polymers, the frictional forces were studied under various normal loads on the $\mathrm{nN}$ and $\mathrm{N}$ scales. Stribeck curves for the most promising polymer, P12MA, were generated on the $\mathrm{N}$ scale, to identify the different lubrication regimes. The following three cases were studied: (i) unmodified silicon ball and wafer (control, both scales), (ii) bare ball and surface grafted with polymers (Figs. 4, 5) (nN scale), and (iii) both surfaces grafted with polymers (Fig. 7) ( $\mathrm{N}$ scale).

\subsection{Nanoscale Friction Measurements}

Nanoscale tribological measurements were carried out by means of AFM. Friction force was recorded at an applied

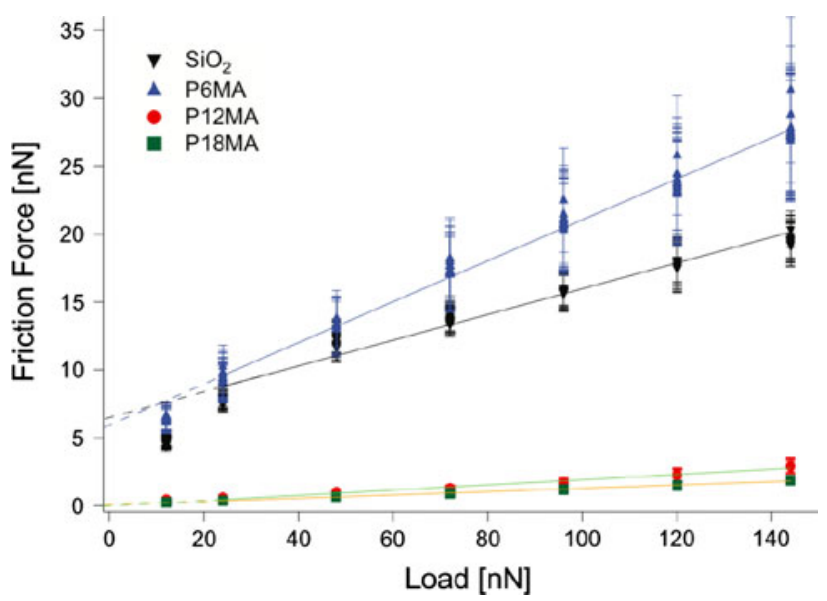

Fig. 4 Friction force as a function of applied load for an unmodified silica colloidal sphere ca. $15 \mu \mathrm{m}$ in diameter) sliding against polymerbrush-functionalized silicon wafers or an unmodified, piranha-cleaned silicon wafer. Measured by LFM in hexadecane. Lines represent the least-mean-square fit of the linear portion of the frictional data. The dashed line is an extrapolation of this linear fit to zero applied load. The coefficient of friction for a given sample is defined as the slope of the linear-fit equation 


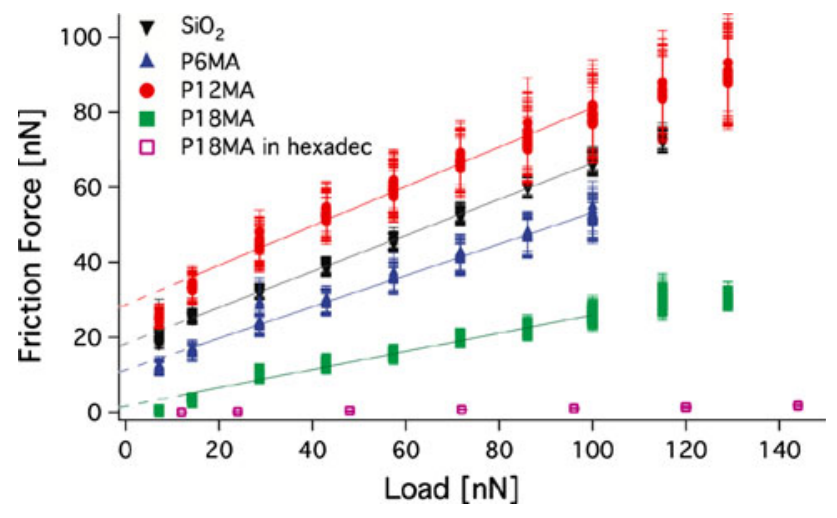

Fig. 5 Friction force as a function of applied load. Unmodified silica colloid sphere sliding against polymer-brush-functionalized silicon wafers or an unmodified, piranha-cleaned silicon wafer. Measured by LFM in ethanol. For reference, a dataset for P18MA in hexadecane was added. Lines represent the least-mean-square fit of the linear portion of the frictional data. The dashed line is an extrapolation of this linear fit to zero applied load. The coefficient of friction for a given sample is defined as the slope of the linear-fit equation

normal load between 10 and $200 \mathrm{nN}$. A least-mean-squareerror linear fit has been performed between the points, in order to obtain reliable coefficient-of-friction values (defined here as the slope of the straight-line friction-load plots).

Figure 4 displays the recorded friction forces as a function of applied normal load, when the bare silica colloid (16 $\mu \mathrm{m}$ in diameter) was slid against an unmodified silicon wafer or polymer-coated wafers, measured in hexadecane. Surfaces coated with P12MA and P18MA display significantly lower coefficients of friction compared to those measured on an unmodified silicon wafer. However, no friction-reducing effect was observed for the poly(hexyl methacrylate)-based brush. For all four substrate types, the friction force increased linearly with increasing load, implying a uniform coefficient of friction over the studied load range. Furthermore, cycles of increasing and decreasing loads for P12MA and P18MA did not reveal any coefficient-of-friction hysteresis, demonstrating the stability of the coatings under these experimental conditions.

The frictional response of the grafted polymers depends on the interaction of the polymers with the surrounding medium $[22,32,33]$. In order to verify the role of the solvent during the friction tests, the same surfaces were studied under ethanol-a liquid in which the free poly(alkyl methacrylates) under investigation were found to be insoluble. The results presented in Fig. 5 show the frictional characteristics for all three polymers, and the bare oxidized silicon wafer, when ethanol was used as a liquid medium. For all four samples, the slopes of their friction-load curves appear comparable. This suggests that under ethanol, similar coefficients of friction were present
Table 1 Coefficient of friction values, obtained from lateral force microscopy

\begin{tabular}{lll}
\hline Sample & COF in hexadecane & COF in ethanol \\
\hline $\mathrm{SiO}_{2}$ & $0.095 \pm 0.001$ & $0.312 \pm 0.148$ \\
$\mathrm{P} 6 \mathrm{MA}$ & $0.151 \pm 0.002$ & $0.463 \pm 0.058$ \\
$\mathrm{P} 12 \mathrm{MA}$ & $0.019 \pm 0.001$ & $0.464 \pm 0.083$ \\
$\mathrm{P} 18 \mathrm{MA}$ & $0.013 \pm 0.001$ & $0.253 \pm 0.018$ \\
\hline
\end{tabular}

A silica-colloid-modified AFM cantilever was used as a countersurface

on all substrates, with some vertical offset on the frictionforce axis, relating to differences in adhesive properties. The coefficient of friction for the polymer-coated wafers is lowest for the P18MA brush with a value of 0.25 , the P6MA and P12MA having COFs of ca. 0.46 . This could be a result of the transition temperatures $T_{\mathrm{g}}$ (and $T_{\mathrm{m}}$ reported for P18MA) of the studied polymers. The higher $T_{\mathrm{g}}$ or high $T_{\mathrm{m}}$ would provide a harder surface at which viscoelastic energy dissipation would be smaller, leading to a lower measured coefficient of friction.

Table 1 presents the calculated coefficient-of-friction values for the LFM-analysed samples in both hexadecane and ethanol. The error bars represent the standard deviation in the coefficient of friction for three measurement locations areas, as obtained from the linear fit.

The frictional data were complemented with an analysis of the adhesive properties of the coatings under different solvent conditions. Adhesive properties were obtained from pull-off forces measured during force-distance curves, performed with a colloid-modified AFM cantilever. A systematic analysis of sets of force-displacement curves has been performed for all samples in ethanol and in hexadecane. The mean adhesion-force values are presented in Fig. 6, and are compared with the coefficient of friction values observed for the given sample.

\subsection{Macroscopic Friction Measurements}

Newton-scale tribological tests were carried out (at $3 \mathrm{~N}$ : 460 MPa maximum Hertzian contact pressure) for the case when both the surfaces were coated with polymers, and compared with a bare-bare silicon-contact control. In these experiments, each surface was grafted with a 250-nm-thick (dry thickness) poly(dodecyl methacrylate) brush. For the case of polymer-coated surfaces, it was possible to maintain a low coefficient of friction for all speeds ranging from $1.5 \mathrm{~cm} / \mathrm{s}$ down to $0.002 \mathrm{~cm} / \mathrm{s}$ during the first run (cumulative distance $2.5 \mathrm{~m}$ ). A repetition without changing the pin position, i.e., following the same wear track, led to coating failure near the end of the second run (cumulative distance $3.6 \mathrm{~m}$ ), observed as an increase in friction coefficient at the $0.75 \mathrm{~cm} / \mathrm{s}$ testing speed (Fig. 7). 
Fig. 6 A comparison of the adhesive forces and coefficientof-friction values obtained in an atomic force microscope. Data collected for studies both in ethanol and hexadecane as a surrounding medium. A silicacolloid-modified AFM cantilever was used as a countersurface
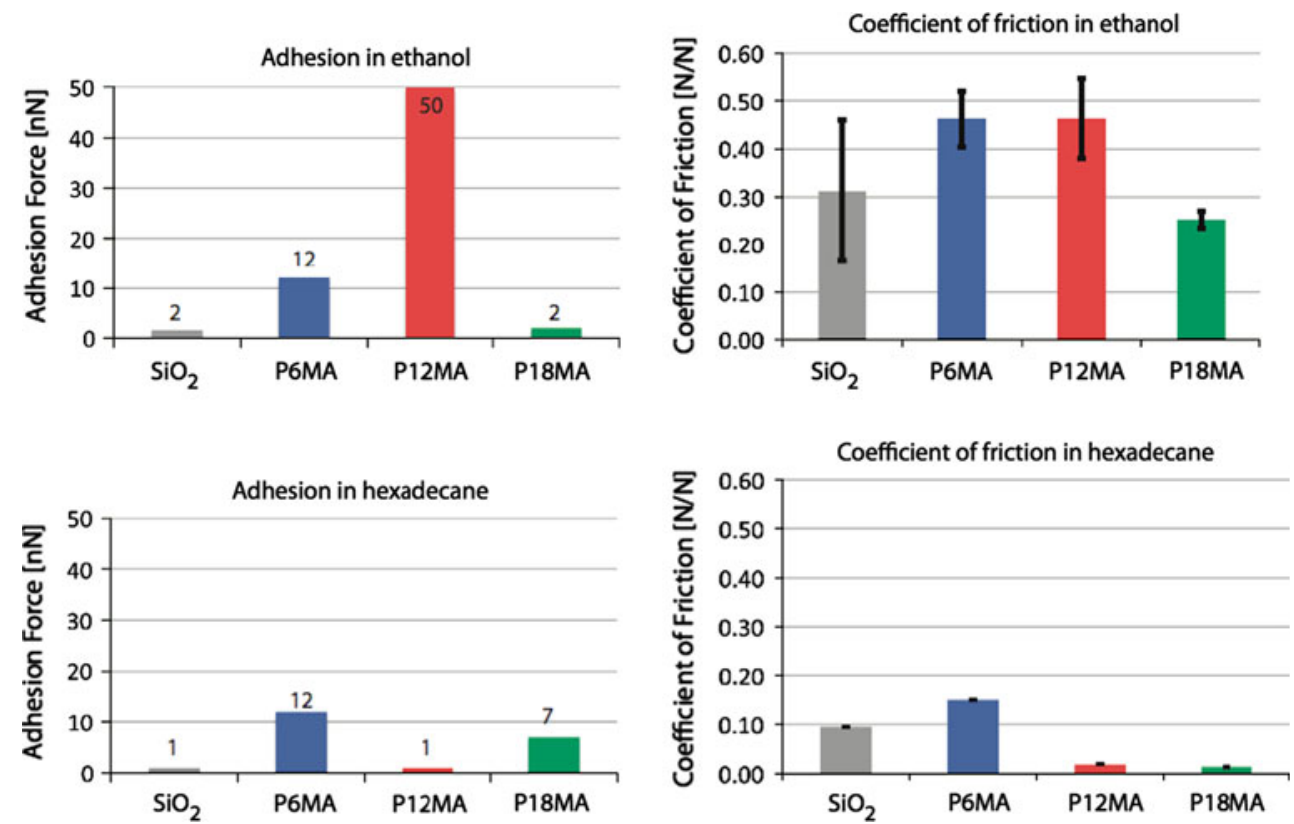

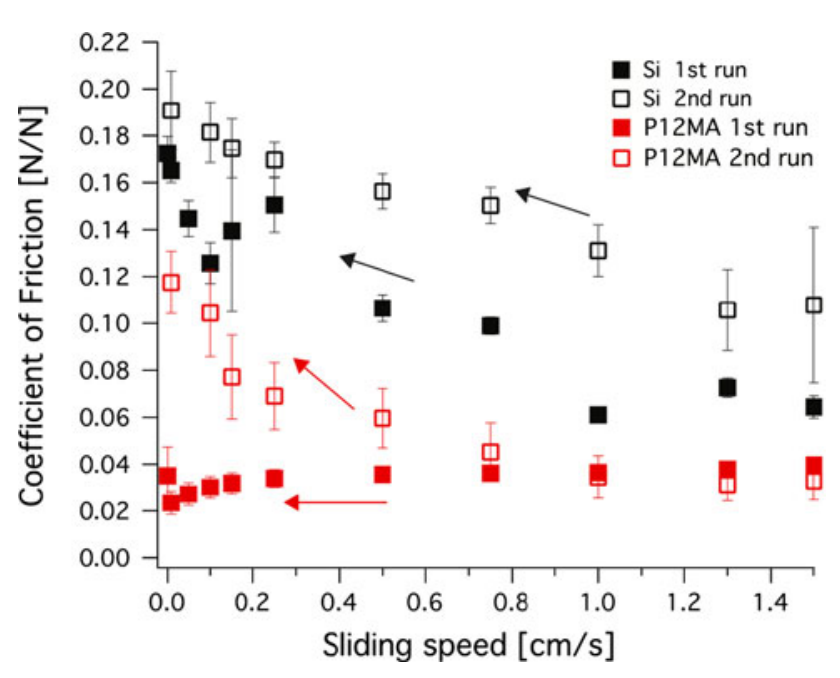

Fig. 7 Brush-brush macrotribological (pin-on-disk) measurements, load $3 \mathrm{~N}\left(p_{\text {avg }}=310 \mathrm{MPa}, p_{\max } 460 \mathrm{MPa}\right)$, radius $1.8 \mathrm{~mm}$, in hexadecane, Si wafer + PLMA $250 \mathrm{~nm}$ (dry thickness), Si ball + PLMA (ca. $250 \mathrm{~nm}$ dry thickness) ca. 20 rotations for each speed. Arrows indicate the direction of speed change during measurement-successively moving from higher to lower values

\section{Discussion}

\subsection{Surface Preparation and Polymerization}

The preparation of poly(octadecyl- dodecyl- and hexylmethacrylates) in solution has been reported, by free-radical polymerization, and, more recently, along with poly(dodecyl acrylate) $[34,35]$ and poly(octadecyl methacrylate) copolymers $[36,37]$ by ATRP. In the present study, SI-ATRP reactions have been carried out as a means to grafting these methacrylate polymers onto surfaces in the form of brushes-previously only carried out for poly(dodecyl acrylate) [38].

The polymerization kinetics are presented in terms of dry polymer thickness. A range of the corresponding molecular weight of the synthesized polymer brushes can be given based on Eq. 1, assuming that the grafting density for SI-ATRP corresponds to that reported in the literature: 0.1-0.7 chains $/ \mathrm{nm}^{2}$ [39, 40]. Grafting densities of 0.5 chains $/ \mathrm{nm}^{2}$ are considered to be the upper limit, and difficult to reach for all but the least bulky monomers, such as methyl methacrylate $\left(0.7\right.$ chains $\left./ \mathrm{nm}^{2}\right)$. On the other hand, grafting densities below $0.05-0.1$ chains $/ \mathrm{nm}^{2}$ are considered to be in the low-density regime achievable by grafting-to strategies [41, 42]. A second indirect method to estimate polymer-brush density [43] assumes that on average 1 out of 10 initiator molecules present in a wellpacked initiator SAM structure is actually employed during the surface-initiated polymerization. Based on Eq. 1, the surface density $\sigma$ of the initiator monolayer (thickness $d=1.8 \mathrm{~nm}, \rho=1.1 \mathrm{~g} / \mathrm{cm}^{3}$, and $M_{\mathrm{w}}=413.89 \mathrm{~g} / \mathrm{mol}$ ) was calculated to be ca. 2.88 initiator $/ \mathrm{nm}^{2}$. Thus, if $10 \%$ of the initiating molecules population would yield a polymer chain, the resulting polymer brush surface density of 0.28 chains $/ \mathrm{nm}^{2}$ would be present at the surface, which is well within the reported $\sigma$ range. Therefore, assuming a chaingrafting density of 0.3 chains $/ \mathrm{nm}^{2}$, the molecular weight for a polymer film of dry thickness 50,150 , and $300 \mathrm{~nm}$ would correspond to $110,000,330,000$, and $660,000 \mathrm{~g} / \mathrm{mol}$, respectively. The assumed grafting density value would be in accordance with the work of Öztürk et al. [38], who have reported poly(dodecyl acrylate) brushes with grafting density $0.27-0.32$ chains $/ \mathrm{nm}^{2}$ and $M_{\mathrm{w}}$ of 44,000 Da after $14 \mathrm{~h}$ of SI-ATRP. Regardless of the rapid kinetics observed 
in our study, the preservation of the living radical character by the ability to reinitiate the brush growth at least twice could still be demonstrated.

$M_{\mathrm{w}}=\frac{d \rho N_{A}}{\sigma}$

The above considerations are strictly theoretical and serve to set the readers' attention on the size scale of the studied polymers. In fact, the real surface grafting density may vary for the three poly(alkyl methacrylates) as a consequence of the different monomer size, which will affect the initiator efficiency due to steric hindrance of the active sites, especially in the case of the larger octadecyl methacrylate molecules. The lower thickness values achieved for the P6MA and P18MA polymers could be related to the lower ATRP catalytic activity of the copper-ligand complex, for which the 12-carbon substituted alkyl methacrylate could be preferred to the other two monomers. Many studies have been conducted on ATRP catalyst structurereactivity relationships, with the important outcome that there exists a need to match a catalyst with a specific monomer to control and exploit the ATRP equilibrium [9].

\subsection{Tribological Response of Hydrophobic Polymer Brushes}

Before dealing with the friction-reducing effect observed in hexadecane for the longer-side-chain poly(alkyl methacrylate)-functionalized surfaces (P12MA and P18MA), the lack of any friction-reducing effect for the polymers studied in ethanol will be discussed. This can be explained in terms of a failure to satisfy the principal requirements needed to form a polymer-brush-based lubricious layer. Highly hydrophobic poly(alkyl methacrylate) brushes are insoluble in polar solvents such as ethanol. This was confirmed empirically for all three polymers and observed as a precipitation of the free polymer from toluene solutions upon addition of ethanol and a swelling ratio of polymer brushes in ethanol close to 1 for P6MA and P12MA brushes (swollen thickness/dry thickness $\approx 1$ ). As a consequence, the brush conformation cannot be attained, and thus sliding only occurs on a collapsed polymeric material. Figure 8 depicts this as being analogous to a bare surface contact when lowering the sliding speed or increasing the load, at which point film failure and asperity contact occurs. A similar explanation can be applied to the high coefficient of friction observed for the P6MA polymer studied in hexadecane, since this polymer is not very soluble in the long-chain hydrocarbon. The adhesive response of poly(hexyl methacrylate) brushes does not vary strongly with a change of the solvent between ethanol and hexadecane, and similarly, the frictional response is similar to that of a bare silicon wafer. This suggests that the P6MA

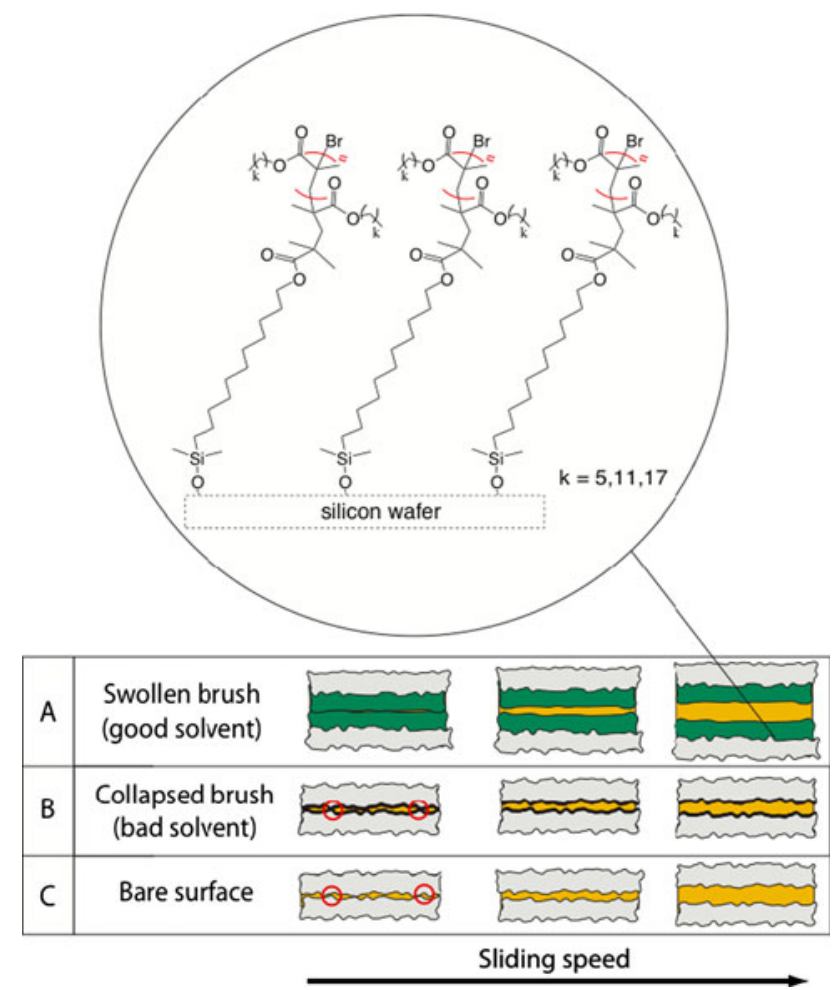

Fig. 8 Schematic representation of polymer-brush-aided lubrication. At lower speeds, the polymer brush prevents asperity-asperity contact and confines the lubricant in the contact area. Snapshots of two sliding bodies with different surface functionalities observed at various speeds are depicted. a Surface functionalized with a polymer brush surface in a good solvent. b Surface functionalized with a polymer brush present in a bad solvent. Collapsed brush-collapsed brush asperity contacts circled at the lower speed. c Bare surfaces in a lubricating fluid. Asperity contacts circled at the lower speed

brush does not react to a solvent change and knowing its incompatibility with ethanol it may be assumed that it is not in a stretched-out brush configuration in hexadecane either. Thus, achievement of low coefficient of friction for P6MA in hexadecane was not observed.

For poly(alkyl methacrylate)s in ethanol, assuming that no extended brush structure is formed and that the chains are poorly solvated, operation under conditions analogous to dry sliding occur. Under these conditions, polymer properties such as glass-transition temperature, roughness, etc., are of importance in determining the friction. Nomura et al. have investigated polystyrene brushes $\left(T_{\mathrm{g}}=100{ }^{\circ} \mathrm{C}\right)$ in a mixtures of toluene (good solvent) and isopropanol (bad solvent), and observed that at high alcohol content a loss of lubricity occurs, attributed to the collapsed, glassy state of the brush [32, 41]. Studies of poly(alkyl methacrylates) have shown that increasing the side-chain length of the alkyl methacrylates generally lowers the $T_{\mathrm{g}}$. The glasstransition temperatures, as measured for bulk polymers, can be approximated for the 6-, 12-, and 18-carbon substituted methacrylates as being $-5,-34$, and $-100{ }^{\circ} \mathrm{C}$, 
respectively $[44,45]$. However, further extension of the side chain to more than 12 carbons correlates with increased flexibility, and allows for the formation of crystalline domains in the structure, and for the P18MA a melting temperature $T_{\mathrm{m}}$ of $27{ }^{\circ} \mathrm{C}$ has been reported [46]. It was observed that in a collapsed state of P18MA the coefficient of friction was lower than for the other polymers, most likely due to the stiff crystalline domains and a smaller plowing effect. It should also be borne in mind that the glass temperatures of surface-tethered polymers that have constrained freedom may in fact be higher than those reported for free polymers [47]. An interesting X-ray photon-correlation-spectroscopy study by Akgun et al. [48] of end-grafted and free polystyrene films showed a significant reduction in surface-height fluctuation for the grafted system.

Based on the above, for the polymer brushes in their collapsed state (e.g., immersed in ethanol), the difference between the glass-transition temperature for a specific polymer and the temperature at which the friction measurements were carried out will influence the contact properties, which are determined by the mobility of the polymeric chains at the temperature of the measurements. This assumption was confirmed by the pull-off measurements carried out in ethanol (compare Fig. 6), where the P12MA coating displayed the highest adhesion force $\left(58{ }^{\circ} \mathrm{C}\right.$ above bulk $T_{\mathrm{g}}$ ), exceeding that of P6MA $\left(29^{\circ} \mathrm{C}\right.$ above bulk $T_{\mathrm{g}}$ ) and P18MA $\left(3{ }^{\circ} \mathrm{C}\right.$ above bulk $\left.T_{\mathrm{m}}\right)$, with the latter displaying negligibly small values, most probably due to the low chain flexibility of the poly(octadecyl methacrylate) at room temperature. The powdery appearance of the dried, free P18MA polymer would correlate with its non-adhesive character observed in AFM. In addition to $T_{\mathrm{g}}$ issues, possible surface segregation of brush components (under different solvent conditions) may influence contact mechanics. No clear correlation between pull-off forces and coefficient of friction was found for the polymers immersed in ethanol.

Tribological studies on the $\mathrm{nN}$ scale of P12MA and P18MA polymer brushes immersed in hexadecane revealed a significant reduction of the coefficient of friction by a factor of $>5$. P12MA in hexadecane also exhibited repulsive behavior during a force-distance curve acquisition with a silica probe, and a 20 -fold decrease in the pull-off forces, consistent with a dense, extended polymer-brush conformation [22]. The lubricious properties of P12MA and P18MA are attributed to their solvation by hexadecane. This was also consistent with the dissolution of the corresponding free polymer samples in the same solvent. For the P12MA polymer brush-hexadecane system the swelling ratio was found to be around 9. Low values of the coefficient of friction were observed, when tested on the $\mathrm{nN}$ scale, for single-side polymer-modified contact.
A comparison of pull-off forces (Fig. 6) with the $y$-intercept of the extrapolated linear section of the lateralforce vs. load curve (Fig. 4) shows reasonable agreement between the two sets of experiments, especially for P6MA and P12MA. For the case of P18MA, in hexadecane, the value obtained from LFM experiments is lower than that of the pull-off force, probably due to the embedding of the colloid in the more static pull-off experiment. For the bare surface, the LFM data obtained in hexadecane displays a tendency towards lower lateral forces at the lowest loads, and it is likely that at zero load, adhesion on the bare surface would be negligible, and thus comparable with the pull-off data.

For tribological studies on the Newton scale for a P12MA double-side-coated contact, under $460 \mathrm{MPa}$ maximal Hertzian contact pressure, very low coefficient-offriction values (below 0.04) were found for all speeds and were nearly invariant with sliding speed $(\mathrm{COF}=0.024$ at $0.01 \mathrm{~cm} \mathrm{~s}^{-1}$ ).

The unmodified ( $\mathrm{Si}-\mathrm{Si}$ ) control contact pair was characterized by a $200 \%$ higher COF than the brush-brush functionalized surfaces, even at the highest testing speed. The coefficient of friction gradually increased upon lowering the sliding speed, reaching 0.19 for $0.01 \mathrm{~cm} \mathrm{~s}^{-1}$.

Prolonged sliding of the P12MA-coated sliding pair in hexadecane on the same track led ultimately to an increase in the coefficient of friction associated with wear. The wear processes are most likely to initiate on the ball, which travels the entire sliding distance with the same point-ofcontact, while locations on the surface are only stressed once per revolution. Following the tribological studies over a sliding distance of $3.6 \mathrm{~m}$, the complete removal of the polymeric film from the disk surface was confirmed by optical microscopy images and AFM imaging of the wear track (Fig. 9). Macroscopic tribological studies are often related to asperity-asperity and non-uniform contact,

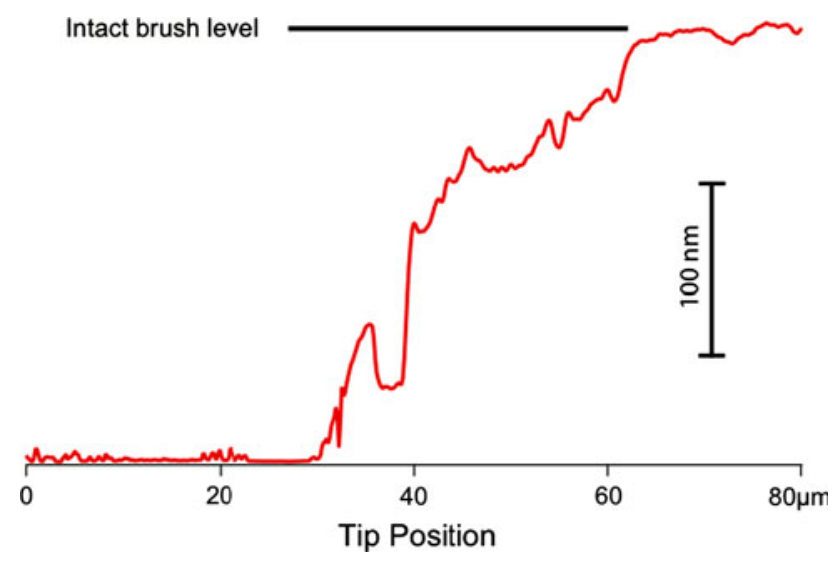

Fig. 9 AFM section of the wear track edge of the P12MA functionalized silicon wafer ( $250 \mathrm{~nm}$ dry thickness) after Newtonscale tribological experiments. Imaging in contact mode in a dry state 
leading to more severe conditions than those predicted by the Hertzian theory. A more detailed exploration of the wear mechanism is the objective of ongoing studies.

\subsection{Conclusions}

Hydrophobic, oil-compatible polymer films have been prepared via a surface-initiated, controlled-radical-polymerization method. Such grafted-from structures are covalently attached to the substrate-thus contributing to their stability, both with respect to the solvent and towards tribological stress. P12MA and P18MA films, were shown on the $\mathrm{nN}$ scale to provide a low coefficient of friction at the level of 0.02 in the presence of a hydrophobic solvent (hexadecane) for brush-hard surface tribological contact in the AFM. However, immersion in a poor solvent for a given polymer brush essentially switched off their lubricous properties. Newton-scale studies of P12MA brushes grafted onto both silicon counterfaces and lubricated with hexadecane withstood over 300 revolutions $(3.6 \mathrm{~m})$ at a maximum Hertzian contact pressure of $460 \mathrm{MPa}$. These results indicate that long alkyl-bearing polymethacrylates, applied here for the first time as lubricants in a brush-like configuration, show great promise as coatings for imparting low friction and protection to metallic and non-metallic sliding partners operating in contact with oils.

\section{References}

1. Hiemenz, P.C., Lodge, T.P.: Polymer chemistry, 2nd edn. CRC Press, Boca Raton (2007)

2. de Gennes, P.G.: Polymers at an interface; a simplified view. Adv. Colloid Interface Sci. 27(3-4), 189-209 (1987)

3. Klein, J.: Shear of polymer brushes. Colloids Surf. A Physicochem. Eng. Aspects 86, 63-76 (1994)

4. Heeb, R., Bielecki, R.M., Lee, S., Spencer, N.D.: Room-temperature, aqueous-phase fabrication of poly(methacrylic acid) brushes by UV-LED-induced, controlled radical polymerization with high selectivity for surface-bound species. Macromolecules 42(22), 9124-9132 (2009)

5. Kobayashi, M., Terayama, Y., Hosaka, N., Kaido, M., Suzuki, A., Yamada, N., Torikai, N., Ishihara, K., Takahara, A.: Friction behavior of high-density poly(2-methacryloyloxyethyl phosphorylcholine) brush in aqueous media. Soft Matter 3, 740-746 (2007)

6. Müller, M., Lee, S., Spikes, H.A., Spencer, N.D.: The influence of molecular architecture on the macroscopic lubrication properties of the brush-like co-polyelectrolyte poly(L-lysine)-g-poly(ethylene glycol) (PLL-g-PEG) adsorbed on oxide surfaces. Tribol. Lett. 15(4), 395-405 (2003)

7. Klein, J.: Shear, friction, and lubrication forces between polymerbearing surfaces. Annu. Rev. Mater. Sci. 26(1), 581-612 (1996)

8. Irfachsyad, D., Tildesley, D., Malfreyt, P.: Dissipative particle dynamics simulation of grafted polymer brushes under shear. Phys. Chem. Chem. Phys. 4(13), 3008-3015 (2002)
9. Braunecker, W.A., Matyjaszewski, K.: Controlled/living radical polymerization: features, developments, and perspectives. Prog. Polym. Sci. 32(1), 93-146 (2007)

10. Edmondson, S., Armes, S.P.: Synthesis of surface-initiated polymer brushes using macro-initiators. Polym. Int. 58(3), 307-316 (2009)

11. Tsujii, Y., Nomura, A., Okayasu, K., Gao, W., Ohno, K., Fukuda, T.: AFM studies on microtribology of concentrated polymer brushes in solvents. J. Phys.: Conf. Ser. 184(1), 012031 (2009)

12. Sakata, H., Kobayashi, M., Otsuka, H., Takahara, A.: Tribological properties of poly(methyl methacrylate) brushes prepared by surface-initiated atom transfer radical polymerization. Polym. J. 37(10), 767-775 (2005)

13. Sanjuan, S., Perrin, P., Pantoustier, N., Tran, Y.: Synthesis and swelling behavior of $\mathrm{pH}$-responsive polybase brushes. Langmuir 23, 5769-5778 (2007)

14. Coessens, V., Pintauer, T., Matyjaszewski, K.: Functional polymers by atom transfer radical polymerization. Prog. Polym. Sci. 26(3), 337-377 (2001)

15. Matyjaszewski, K., Miller, P.J., Shukla, N., Immaraporn, B., Gelman, A., Luokala, B.B., Siclovan, T.M., Kickelbick, G., Vallant, T., Hoffmann, H., Pakula, T.: Polymers at interfaces: using atom transfer radical polymerization in the controlled growth of homopolymers and block copolymers from silicon surfaces in the absence of untethered sacrificial initiator. Macromolecules 32(26), 8716-8724 (1999)

16. Street, G., Illsley, D., Holder, S.: Optimization of the synthesis of poly(octadecyl acrylate) by atom transfer radical polymerization and the preparation of all comblike amphiphilic diblock copolymers. J. Polym. Sci.: Polym. Chem. 43(5), 1129-1143 (2005)

17. Bergenudd, H., Coullerez, G., Jonsson, M., Malmström, E.: Solvent effects on ATRP of oligo(ethylene glycol) methacrylate. Exploring the limits of control. Macromolecules 42(9), 33023308 (2009)

18. Patten, T.E., Matyjaszewski, K.: Atom transfer radical polymerization and the synthesis of polymeric materials. Adv. Mater. 10(12), 901-915 (1998)

19. Li, A., Benetti, E.M., Tranchida, D., Clasohm, J.N., Schönherr, H., Spencer, N.D.: Surface-grafted, covalently cross-linked hydrogel brushes with tunable interfacial and bulk properties. Macromolecules 44(13), 5344-5351 (2011)

20. Kobayashi, M., Takahara, A.: Tribological properties of hydrophilic polymer brushes under wet conditions. Chem. Rec. 10(4), 208-216 (2010)

21. Barbey, R., Klok, H.: Room temperature, aqueous post-polymerization modification of glycidyl methacrylate-containing polymer brushes prepared via surface-initiated atom transfer radical polymerization. Langmuir 26(23), 18219-18230 (2010)

22. Sui, X., Zapotoczny, S., Benetti, E.M., Schon, P., Vancso, G.J.: Characterization and molecular engineering of surface-grafted polymer brushes across the length scales by atomic force microscopy. J. Mater. Chem. 20(24), 4981-4993 (2010)

23. Fan, J., Müller, M., Stöhr, T., Spikes, H.: Reduction of friction by functionalised viscosity index improvers. Tribol. Lett. 28(3), 287-298 (2007)

24. Lavanant, L., Pullin, B., Hubbell, J.A., Klok, H.-A.: A facile strategy for the modification of polyethylene substrates with nonfouling, bioactive poly(poly(ethylene glycol) methacrylate) brushes. Macromol. Biosci. 10(1), 101-108 (2010)

25. Tugulu, S., Arnold, A., Sielaff, I., Johnsson, K., Klok, H.-A.: Protein-functionalized polymer brushes. Biomacromolecules 6(3), 1602-1607 (2005)

26. Xu, W., Zhu, X., Cheng, Z., Chen, J.: Atom transfer radical polymerization of lauryl methacrylate. J. Appl. Polym. Sci. 90(4), 1117-1125 (2003) 
27. Ducker, W.A., Senden, T.J., Pashley, R.M.: Direct measurement of colloidal forces using an atomic force microscope. Nature $\mathbf{3 5 3}$, 239-241 (1991)

28. Cannara, R.J., Eglin, M., Carpick, R.W.: Lateral force calibration in atomic force microscopy: a new lateral force calibration method and general guidelines for optimization. Rev. Sci. Instrum. 77(053701), 1-11 (2006)

29. Jones, D.M., Brown, A.A., Huck, W.T.S.: Surface-initiated polymerizations in aqueous media: effect of initiator density. Langmuir 18(4), 1265-1269 (2002)

30. Laibinis, P.E., Whitesides, G.M., Allara, D.L., Tao, Y.T., Parikh, A.N., Nuzzo, R.G.: Comparison of the structures and wetting properties of self-assembled monolayers of $n$-alkanethiols on the coinage metal surfaces, copper, silver, and gold. J. Am. Chem. Soc. 113(19), 7152-7167 (1991)

31. Liu, G., Yan, L., Chen, X., Zhang, G.: Study of the kinetics of mushroom-to-brush transition of charged polymer chains. Polymer 47(9), 3157-3163 (2006)

32. Nomura, A., Okayasu, K., Ohno, K., Fukuda, T., Tsujii, Y.: Lubrication mechanism of concentrated polymer brushes in solvents: effect of solvent quality and thereby swelling state. Macromolecules 44(12), 5013-5019 (2011)

33. Heeb, R., Lee, S., Venkataraman, N.V., Spencer, N.D.: Influence of salt on the aqueous lubrication properties of end-grafted, ethylene glycol-based self-assembled monolayers. ACS Appl. Mater. Interfaces 1(5), 1105-1112 (2009)

34. Beers, K.L., Matyjaszewski, K.: The atom transfer radical polymerization of lauryl acrylate. J. Macromol. Sci. Pure Appl. Chem. A 38(7), 731-739 (2001)

35. Liénafa, L., Monge, S., Robin, J.-J.: A versatile synthesis of poly(lauryl acrylate) using $\mathrm{N}$-(n-octyl)-2-pyridylmethanimine in copper mediated living radical polymerization. Eur. Polym. J. 45(6), 1845-1850 (2009)

36. Qin, S., Saget, J., Pyun, J., Jia, S., Kowalewski, T.: Synthesis of block, statistical, and gradient copolymers from octadecyl (meth)acrylates using atom transfer radical polymerization. Macromolecules 36(24), 8969-8977 (2003)

37. Neugebauer, D., Theis, M., Pakula, T., Wegner, G., Matyjaszewski, K.: Densely heterografted brush macromolecules with crystallizable grafts. Synthesis and bulk properties. Macromolecules 39(2), 584-593 (2005)

38. Öztürk, E., Turan, E., Caykara, T.: Fabrication of ultrahydrophobic poly(lauryl acrylate) brushes on silicon wafer via surfaceinitiated atom transfer radical polymerization. Appl. Surf. Sci. 257(3), 1015-1020 (2010)
39. Kobayashi, M., Yamaguchi, H., Terayama, Y., Wang, Z., Ishihara, K., Hino, M., Takahara, A.: Structure and surface properties of high-density polyelectrolyte brushes at the interface of aqueous solution. Macromol. Symp. 279(1), 79-87 (2009)

40. Terayama, Y., Kikuchi, M., Kobayashi, M., Takahara, A.: Welldefined poly(sulfobetaine) brushes prepared by surface-initiated ATRP using a fluoroalcohol and ionic liquids as the solvents. Macromolecules 44(1), 104-111 (2011)

41. Lego, B., Skene, W.G., Giasson, S.: Swelling study of responsive polyelectrolyte brushes grafted from mica substrates: effect of $\mathrm{pH}$, salt, and grafting density. Macromolecules 43(9), 4384-4393 (2010)

42. Nomura, A., Okayasu, K., Ohno, K., Fukuda, T., Tsujii, Y.: Lubrication mechanism of concentrated polymer brushes in solvents: effect of solvent quality and thereby swelling state. Macromolecules 44, 5013-5019 (2011)

43. Ma, H., He, J., Liu, X., Gan, J., Jin, G., Zhou, J.: Surface initiated polymerization from substrates of low initiator density and its applications in biosensors. ACS Appl. Mater. Interfaces 2(11), 3223-3230 (2010)

44. Dudognon, E., Bernès, A., Lacabanne, C.: Nature of molecular mobility through the glass transition in poly(n-alkyl methacrylates): a study by dielectric spectroscopies. J. Macromol. Sci. B 43(3), 591-604 (2005)

45. Çayli, G., Meier, M.: Polymers from renewable resources: bulk ATRP of fatty alcohol-derived methacrylates. Eur. J. Lipid Sci. Technol. 110(9), 853-859 (2008)

46. Hempel, E., Huth, H., Beiner, M.: Interrelation between side chain crystallization and dynamic glass transitions in higher poly(n-alkyl methacrylates). Thermochim. Acta 403, 105-114 (2003)

47. Savin, D., Pyun, J., Patterson, G., Kowalewski, T., Matyjaszewski, K.: Synthesis and characterization of silica-graft-polystyrene hybrid nanoparticles: effect of constraint on the glass-transition temperature of spherical polymer brushes. J. Polym. Sci. B: Polym. Phys. 40(23), 2667-2676 (2002)

48. Akgun, B., Uğur, G., Jiang, Z., Narayanan, S., Song, S., Lee, H., Brittain, W.J., Kim, H., Sinha, S.K., Foster, M.D.: Surface dynamics of "dry" homopolymer brushes. Macromolecules 42(3), 737-741 (2009) 